\title{
Association of Household Opioid Availability and Prescription Opioid Initiation Among Household Members
}

Marissa J. Seamans, PhD; Timothy S. Carey, MD, MPH; Daniel J. Westreich, PhD; Stephen R. Cole, PhD; Stephanie B. Wheeler, PhD; G. Caleb Alexander, MD, MS; Virginia Pate, MS; M. Alan Brookhart, PhD

IMPORTANCE Increases in prescription opioid use in the United States have been attributed to changing prescribing guidelines and attitudes toward pain relief; however, the spread of opioid use within households through drug diversion may also be a contributing factor.

OBJECTIVE To investigate whether individuals living in a household with a prescription opioid user are more likely to initiate prescription opioids themselves, compared with individuals in households with a prescription nonsteroidal anti-inflammatory drug (NSAID) user.

DESIGN, SETTING, AND PARTICIPANTS This was a retrospective cohort study using administrative health care claims data from 2000 to 2014 of commercial insurance beneficiaries sharing a health plan with continuous prescription drug coverage, without opioid or NSAID use in the prior year. Enrollees were followed from the date of the first prescription filled by a household member for an eligible opioid or NSAID until initiation of prescription opioids, disenrollment, or administrative censoring after 1 year or the end of follow-up on December 31, 2014. Risk of opioid initiation was derived from inverse probability-weighted (IPW) Kaplan-Meier estimators that adjusted for potential confounders, prognostic factors, and predictors of censoring.

EXPOSURE Outpatient pharmacy dispensing of a prescription opioid or prescription NSAID.

MAIN OUTCOMES AND MEASURES Incident outpatient pharmacy fill for a prescription opioid by a household member.

RESULTS From 2000 to 2014, 12695280 individuals were exposed to prescription opioids and 6359639 to prescription NSAIDS through an index prescription to a household member. The IPW estimated risk of opioid initiation in the subsequent year was $11.83 \%(95 \% \mathrm{Cl}$, $11.81 \%-11.85 \%$ ) among individuals exposed to prescription opioids in the household, compared with $11.11 \%$ ( $95 \% \mathrm{Cl}, 11.09 \%-11.14 \%$ ) among individuals exposed to prescription NSAIDs, resulting in a risk difference of $0.71 \%(95 \% \mathrm{Cl}, 0.68 \%-0.74 \%)$. An unmeasured confounder that is modestly associated with the exposure (eg, prevalence difference $=0.9 \%$ ) and the outcome (eg, risk difference $=0.9$ ) after adjustment for all measured variables could explain our observed estimate of the overall risk difference $(0.71 \%)$.

CONCLUSIONS AND RELEVANCE Living in a household with a prescription opioid user may increase risk of prescription opioid use, which may reflect both increased access to these products as well as shared risk factors, such as prescriber preference and prescription drug monitoring.

\footnotetext{
Author Affiliations: Department of Epidemiology, Gillings School of Global Public Health, University of North Carolina at Chapel Hill (Seamans, Carey, Westreich, Cole, Pate, Brookhart); Division of General Medicine \& Clinical Epidemiology, University of North Carolina at Chapel Hill School of Medicine (Carey); Department of Health Policy, Gillings School of Global Public Health, University of North Carolina at Chapel Hill (Wheeler); Center for Drug Safety and Effectiveness, Johns Hopkins University, Baltimore, Maryland (Alexander); Department of Epidemiology, Johns Hopkins Bloomberg School of Public Health, Johns Hopkins University, Baltimore, Maryland (Alexander).

Corresponding Author: Marissa J. Seamans, PhD, Department of Mental Health, Johns Hopkins University Bloomberg School of Public Health, 624 N Broadway, Baltimore, MD 21205 (seamans@jhu.edu).
} 
O pioid prescribing in the US increased 300\% from 1991 to 2009 and totaled 246 million dispensed prescriptions in 2015. ${ }^{1-3}$ Globally, the United States continues to be the largest consumer of the world's supply of hydrocodone (99\%) and oxycodone (81\%), ${ }^{4}$ with hydrocodone-acetaminophen being the leading prescription drug dispensed by US retail pharmacies. ${ }^{5}$ This increase in opioid prescribing has been attributed to changes in prescribing guidelines, ${ }^{6}$ attitudes toward pain relief, ${ }^{7}$ aggressive pharmaceutical marketing, ${ }^{8}$ and the liberalization of laws governing the ability of physicians to prescribe opioids without training in pain management. ${ }^{9}$ Opioid adverse effects include QT interval prolongation and respiratory depression, which can lead to opioid-related emergency department visits, ${ }^{10,11}$ hospitalizations, ${ }^{12}$ and death. ${ }^{13}$ In 2011, prescription opioids were involved in more than 480000 ER visits and 16000 deaths, surpassing the mortality burden from firearms and motor vehicle accidents for Americans ages 35 to 54 years. ${ }^{14}$ Accidental ingestion of prescription opioids also caused more than 5000 ER visits among children 5 years or younger in 2011, which underscores the harms of the broad availability of opioids in US households. ${ }^{15}$

Opioids are often prescribed in doses exceeding clinical guidelines for patients with non-cancer-related pain, ${ }^{16}$ and in large quantities, resulting in surpluses of opioids stored in household medicine cabinets. ${ }^{17}$ Unused medications create opportunities for nonprescribed use and drug sharing among friends and family members, who may perceive these medications to be low risk given their storage at home. ${ }^{18}$ For example, a third of veterans receiving prescription opioid therapy report sharing unused medications with family members, ${ }^{18}$ and more than $70 \%$ of non-medical opioid users obtain the drugs from family members and friends. ${ }^{19,20}$ Because prescription medication sharing is common, ${ }^{21} \mathrm{fami}$ lies and other social networks likely shape norms and behaviors surrounding the use of prescription opioids. Given the documented spread of substance use behaviors, such as heavy drinking within social networks, ${ }^{22-25}$ it is likely that prescription opioid use can also spread within networks, such as families. Although a previous study has examined the association between opioid abuse in one person and opioid abuse in another, ${ }^{26}$ the extent to which prescribed opioid use in one person is associated with increased risk of prescribed opioid use in another person remains unknown.

We quantified the association between potential access to opioids resulting from a new prescription started by a household member and subsequent new use of prescription opioids by other family members. Specifically, we compared the 1-year risk of opioid initiation due to the introduction of prescription opioids vs introduction of nonopioid analgesics (prescription nonsteroidal anti-inflammatory drugs [NSAIDs]) in households of commercial insurance beneficiaries that included employees, spouses, and dependents (hereinafter, opioid households and NSAID households). We further explored whether associations varied across subgroups of age, geographic region, potential indications, refill availability, days' supply, and by year and opioid dose.

\section{Key Points}

Question Is prescription opioid use in one household member associated with increased risk of prescribed opioid use in other household members?

Findings In a study comparing 12695280 commercial insurance beneficiaries with a household member who started a new prescription of opioids, to 6359639 beneficiaries with a household member who started a new prescription of nonopioid pain relievers, the 1 -year risk of subsequent opioid use was $0.71 \%$ higher among individuals exposed to opioids through a household member's prescription.

Meaning Prescription opioid use may spread within households.

\section{Methods}

We used the 2000-2014 Truven Health Analytics MarketScan Commercial Claims and Encounters databases covering the years 2000 to 2014. MarketScan contains standardized, deidentified, person-level information on enrollment, paid inpatient and outpatient services and procedures, and outpatient pharmacy dispensing claims of employer-sponsored commercial insurance beneficiaries, their spouses, and dependents. MarketScan is one of the largest, fully integrated, and most complete commercial insurance claims databases available for the United States, where beneficiaries can be tracked across insurance plans, health care sites, clinician types, and over time ${ }^{27}$ with median follow-up of 3 years. The database increased from 3.7 million enrollees to 47.2 million from 2000 to 2014. The Office of Human Research Ethics at the University of North Carolina at Chapel Hill deemed the study exempt from review.

\section{Study Population}

This retrospective cohort study included household members of patients who initiated use of prescription opioids or prescription NSAIDs based on outpatient pharmacy dispensing claims (henceforth, we refer to the index patient as "Patient 0 "). Prescription NSAIDs were chosen as the active comparator group because they have similar indications for treating pain, which minimizes the potential for unmeasured confounding. New use was defined as the first pharmacy dispensing claim after a 365-day period of continuous enrollment without evidence of prescription opioid or prescription NSAID use in claims. We generalized the "new-user" design ${ }^{28}$ to "new households" by requiring all household members to have continuous prescription drug coverage with no record of prescription opioid or NSAID use during the baseline period. Household members entered either the opioid or NSAID cohort at the index date anchored to the date of initiation by Patient 0. Eligible index dates were those occurring between January 1, 2001, and December 31, 2014, to ensure observation of the 1-year baseline. To make comparisons between cohorts more rigorous and identify index patients with similar indications, household members of patients who had a diagnosis of malignant neoplasm, used hospice services during the baseline 
period, or initiated both prescription opioids and NSAIDs on the same day were excluded.

\section{Opioid Medication Exposure}

Pharmacy dispensing billing claims for the most commonly prescribed synthetic and semisynthetic opioids and NSAIDs were identified in the Outpatient Pharmaceutical Claims file by their generic string name. Opioids and NSAIDs were restricted to oral and transdermal formulations (see eTable 1 in the Supplement). We excluded claims for opioids used primarily to treat Parkinson disease (apomorphine), opioid dependence (methadone), and cough (potassium guaiacolsulfonate-hydrocodone bitartrate).

\section{Outcome Assessment}

Our primary outcome was initiation of prescription opioids by members of theindex patient's household. Initiation by a household member was assessed similarly to cohort eligibility defined by Patient $\mathrm{O}$ using the dispensing date for an eligible opioid in the Outpatient Pharmaceutical claims database.

\section{Covariates}

Baseline covariates for household members were assessed during the year prior to the index date defined by Patient 0. Potential confounders of the association between opioid receipt in Patient 0 and opioid initiation by household members were identified a priori using subject matter knowledge and causal diagrams, ${ }^{29}$ and included household size, composition (children $<18$ years, yes/no), region of residence (Northeast, North Central, South, West), calendar year of cohort entry, and history of substance use diagnosis for Patient 0 (yes/ no). Baseline predictors of loss to follow-up (defined as disenrollment) and opioid initiation of at-risk household members included age, sex, history of chronic pain diagnosis, psychiatric comorbidity (dichotomous variables for yes/no), health care utilization (number of outpatient visits, continuous; emergency department visit in prior 7 or 30 days, yes/no), and use of scheduled prescription medications (dichotomous variables for yes/no for each drug class; see eTable 1 in the Supplement for definitions).

\section{Statistical Analyses}

We estimated the 1-year risk of opioid initiation by household members (excluding Patients 0 ) within each treatment group using the complement of the Kaplan-Meier estimator with time in days from the index date. Household members were individually censored at the earliest of an event, at 1 year of followup, disenrollment, or administrative censoring on December 31, 2014.

We estimated 1-year risks and risk differences (RDs) for opioid initiation using inverse probability-weighted KaplanMeier estimators adjusting for baseline confounders, prognostic factors, and dropout. ${ }^{30}$ Weights were the product of inverse probability of treatment and inverse probability of censoring weights (see the eMethods in the Supplement for details on the construction of weights). Age, number of outpatient visits during baseline, and household size were modeled using restricted quadratic splines ${ }^{31}$ and calendar year as a cat- egorical variable with each year as a separate category. Estimates of precision were obtained using nonparametric bootstrap resampling with replacement.

We explored potential heterogeneity of the association between household opioid availability and opioid initiation across subgroups: age of at-risk household members (0-5, 6-11, 12$17,18-25,26-35,36-45,46-55$, $\geq 56$ years); region of residence; potential indication for Patients 0 (back pain [yes vs no]; fracture [yes vs no]; year of cohort entry; and characteristics of the index prescription (availability of refills [yes vs no]; days of supply [ $<30$ days or $\geq 30$ days]). Subgroup differences were examined by stratifying the original cohort and repeating the primary analysis described herein within each stratum. We further examined variations in risk by daily opioid dose by converting all index opioid prescriptions to morphine milligrams equivalents (MME) and categorized dosage as 0 to 19, 20 to 50, 51 to 89 , or 90 or more MME/d. ${ }^{32,33}$

We performed 2 sensitivity analyses. First, the primary analysis was performed after restricting to 2-adult (age $>18$ years) households to account for shared genetic factors that could give rise to opioid-seeking behavior. Second, we assessed the extent to which unmeasured differences between the opioid and NSAID cohorts could explain our results. ${ }^{34,35}$ Additional details are provided in the eMethods in the Supplement. All analyses were conducted using SAS statistical software (version 9.4; SAS Institute Inc).

\section{Results}

\section{Characteristics of the Study Sample}

The study sample comprised 12695280 members of 5871003 opioid households and 6359639 members of 3015932 NSAID households, after excluding 84810 households (1\%) without information on geographic region (Figure 1). Opioid and NSAID households were of similar size (median, 3 enrollees; interquartile range [IQR], 2-4 enrollees), but opioid households were more likely to reside in the South than NSAID households (Table 1). Demographic characteristics were similar between cohorts: the median age of household members was 22 years (IQR, 11-45 years), and less than half were female. Overall, baseline covariates were balanced between household exposure groups. However, compared with members of NSAID households, slightly greater proportions of members of opioid households had used attention-deficit/hyperactivity disorder medications, antibiotics, benzodiazepines, selective serotonin reuptake inhibitors (SSRIs), and sleep medications, but not $\beta$-blockers, statins, or muscle relaxants during baseline. Members of opioid households were also more likely than members of NSAID households to have a history of back and neck pain, migraine, fractures, depression, substance abuse, and cancer screening, and less likely to have diabetes mellitus and arthritis. However, utilization of outpatient and inpatient services and emergency department visits were similar between household exposure groups.

\section{Unadjusted Analysis}

Members of opioid households and NSAID households were followed for a median of 1 year (IQR, 0.6-1.0-year). During 
Figure 1. Derivation of Cohort in the MarketScan Commercial Claims and Encounters Databases, 2000-2014

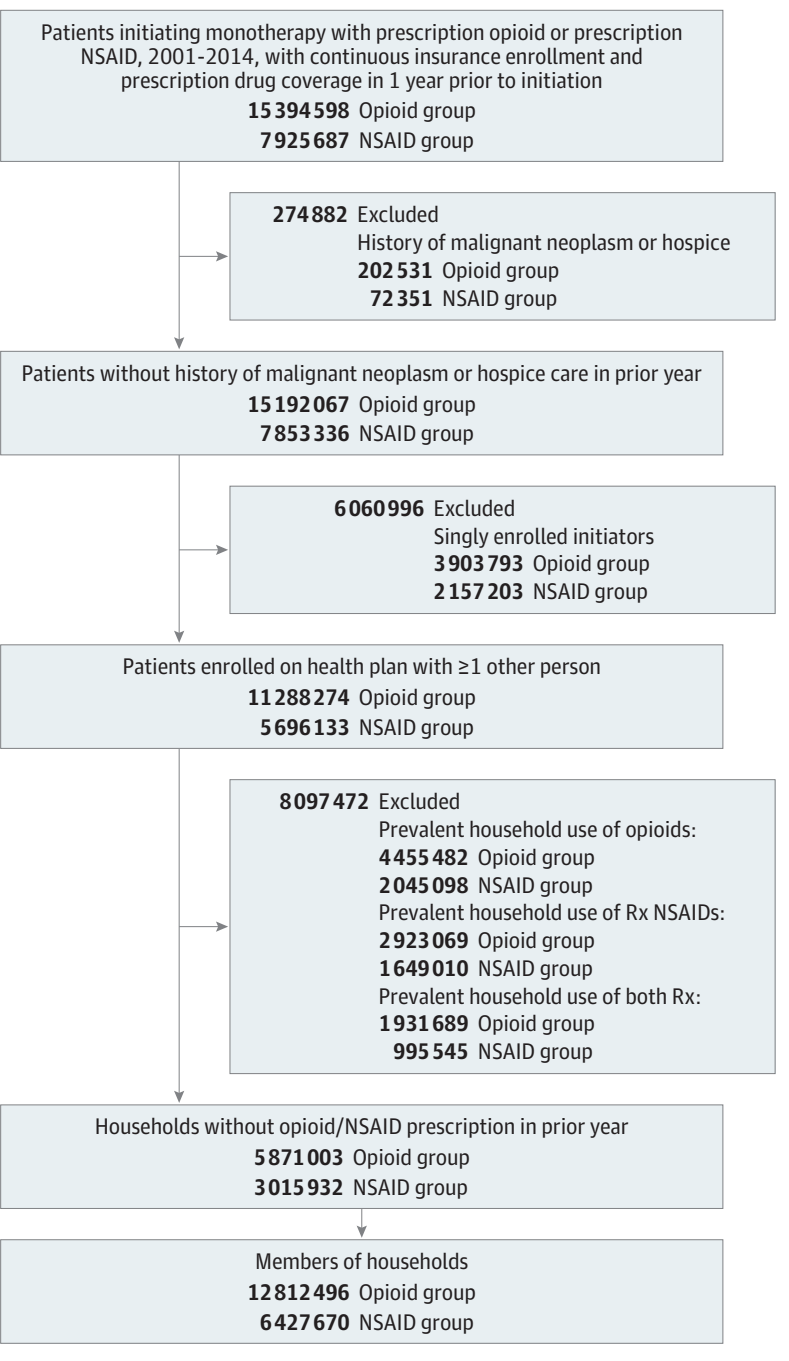

NSAID indicates nonsteroidal anti-inflammatory drug; Rx, prescription

14846450 person-years of follow-up, 1786014 individuals initiated use of prescription opioids and 4187048 individuals disenrolled from their health plan (21\% in NSAID households, $22 \%$ in opioid households). Overall, the 1-year risk of prescription opioid initiation by a second household member was $11.32 \%$ (95\% CI, 11.30\%-11.34\%). The 1-year unadjusted risk of prescription opioid initiation was $11.68 \%$ (95\% CI, $11.66 \%-$ $11.70 \%$ ) within opioid households and $10.60 \%$ (95\% CI, 10.57\%10.63\%) within NSAID households.

Inverse Probability-Weighted Analysis

All potential confounders and predictors of opioid initiation or dropout identified a priori were included in corresponding propensity score models for treatment and censoring weights. The means of the treatment and censoring weights were 1.00 (range, 0.36-6.06) and 0.98 (range, 0.36-3.59), respectively. Cumulative incidence curves for opioid initiation over 1 year of follow-up by household opioid availability are presented in
Table 1. Baseline Characteristics of Household Members of Prescription Analgesic Initiators, MarketScan Commercial Claims and Encounters, 2000-2014

\begin{tabular}{|c|c|c|}
\hline Characteristic & $\begin{array}{l}\text { Opioid Households } \\
(\mathrm{n}=12695280)\end{array}$ & $\begin{array}{l}\text { NSAID Households } \\
(\mathrm{n}=6359639)\end{array}$ \\
\hline \multicolumn{3}{|l|}{ Demographics } \\
\hline Female sex & 48.5 & 48.1 \\
\hline Age, median (IQR), y & $22(11-45)$ & $21(11-45)$ \\
\hline \multicolumn{3}{|l|}{ Age group, y } \\
\hline$<6$ & 10.8 & 10.4 \\
\hline $6-11$ & 15.1 & 15.6 \\
\hline $12-17$ & 15.7 & 16.5 \\
\hline $18-25$ & 11.8 & 13.1 \\
\hline $26-35$ & 7.4 & 6.3 \\
\hline $36-45$ & 14.8 & 13.7 \\
\hline $46-55$ & 16.2 & 15.8 \\
\hline$\geq 56$ & 8.3 & 8.7 \\
\hline \multicolumn{3}{|l|}{ Baseline mediation use } \\
\hline Benzodiazepines & 3.1 & 2.8 \\
\hline ADHD medications & 1.5 & 1.4 \\
\hline Antibiotics & 36.0 & 35.8 \\
\hline Muscle relaxants & 1.0 & 1.0 \\
\hline Sleep medications & 1.8 & 1.6 \\
\hline SSRIS & 4.6 & 4.1 \\
\hline Statins & 6.0 & 6.2 \\
\hline \multicolumn{3}{|l|}{ Preexisting pain conditions } \\
\hline Back and neck pain & 7.6 & 7.0 \\
\hline Back pain & 3.7 & 3.5 \\
\hline Back disorder & 5.9 & 5.7 \\
\hline Headache & 2.3 & 2.4 \\
\hline Migraine & 1.0 & 1.0 \\
\hline Arthritis & 6.3 & 6.3 \\
\hline Osteoarthritis & 0.9 & 0.9 \\
\hline Rheumatoid arthritis & 0.4 & 0.4 \\
\hline Fibromyalgia & 0.9 & 0.8 \\
\hline Fractures & 1.7 & 1.7 \\
\hline \multicolumn{3}{|l|}{ Preexisting comorbidities } \\
\hline Depression & 2.8 & 2.6 \\
\hline Substance abuse & 1.0 & 1.0 \\
\hline Alcohol use & 0.3 & 0.3 \\
\hline Gastrointestinal bleeding & 0.5 & 0.5 \\
\hline COPD & 0.4 & 0.4 \\
\hline \multicolumn{3}{|l|}{ Health care utilization } \\
\hline ER visit in past $30 \mathrm{~d}$ & 1.1 & 1.1 \\
\hline ER visit in past $7 \mathrm{~d}$ & 0.4 & 0.3 \\
\hline Outpatient visits, mean (SD) & $2.3(2.95)$ & $2.4(3.03)$ \\
\hline Inpatient days, mean (SD) & $0.1(1.40)$ & $0.1(1.42)$ \\
\hline
\end{tabular}

Abbreviations: ADHD, attention-deficit/hyperactivity disorder; COPD, chronic obstructive pulmonary disease; ER, emergency department; IQR, interquartile range; SSRIs, selective serotonin reuptake inhibitors.

a Data are given as percentages except where noted. Covariates are defined in eTable 1 in the Supplement. Baseline characteristics were assessed in the $1 \mathrm{y}$ prior to the index date.

Figure 2. The 1-year risk of opioid initiation was $11.83 \%$ (95\% CI, $11.81 \%-11.85 \%)$ among individuals in an opioid household, compared with $11.11 \%$ (95\% CI, 11.09\%-11.14\%) among 
Figure 2. Inverse Probability-Weighted Cumulative Incidence Curves for Opioid Initiation by Household Availability of Prescription Opioids, MarketScan Commercial Claims and Encounters, 2000-2014

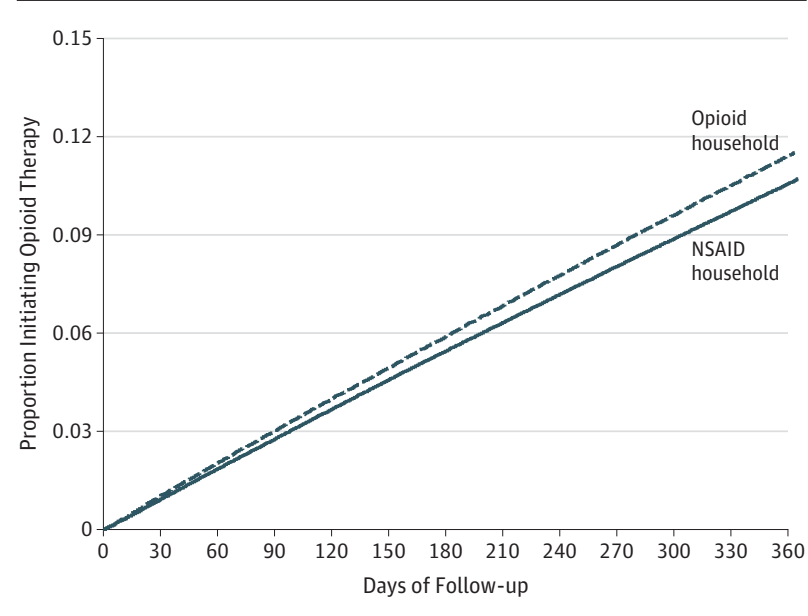

Estimates adjusted for household size, region, year of cohort entry, family history of substance abuse, age, sex, baseline pain and psychiatric co-morbidities, use of scheduled and unscheduled prescription medications, and health care utilization. NSAID indicates nonsteroidal anti-inflammatory drug

individuals in an NSAID household (Table 2). The adjusted 1-year RD in opioid initiation due to potential access to household opioids was $0.71 \%$ (95\% CI, 0.68\%-0.74\%).

\section{Subgroup Analyses}

The association between household opioid availability and opioid initiation varied across subgroups of age, geographic region, and potential indication of Patient $\mathrm{O}$ (Table 2). The RD among younger adults ages 18 to 25 years was $0.91 \%$ (95\% CI, 0.81\%-1.01\%), compared with 1.26\% (95\% CI, 1.08\%-1.43\%) among those ages 26 to 35 years. In the North Central region, the RD was $0.44 \%$ (95\% CI, 0.38\%-0.51\%), compared with 0.95\% (95\% CI, 0.88\%-1.02\%) in the West. Risk differences did not vary by history of back and neck pain in Patient O, but RDs were markedly smaller among household members of patients with a history of fracture (RD, $0.42 \%$ [95\% CI, $0.21 \%$ $0.61 \%]$ ) than those without (RD, $0.82 \%$ [95\% CI, $0.79 \%$ $0.85 \%])$

Results varied across characteristics of the index prescription (Table 3). The RD for opioid initiation was 0.53\% (95\% CI, $0.49 \%-0.57 \%$ ) in households where the index supply was less than 30 days, compared with 1.14\% (95\% CI, 0.97\% $1.34 \%$ ) in households with at least a 30-day supply. The RD was 0.44\% (95\% CI, 0.40\%-0.47\%) in households with a single fill of the index prescription, compared with $1.43 \%$ (95\% CI, 1.33\%$1.53 \%$ ) in households with refills. Compared with NSAID households, the RD for opioid initiation in households with an index opioid prescription for less than $20 \mathrm{MME} / \mathrm{d}$ was $1.44 \%$ (95\% CI, $1.39 \%-1.50 \%$ ), 0.81\% (95\% CI, 0.77\%-0.85\%) for $20-50$ MME/d, 0.78\% (95\% CI, 0.72\%-0.83\%) for 51-89 MME/d, and $1.02 \%$ (95\% CI, 0.95\%-1.07\%) for $\geq 90 \mathrm{MME} / \mathrm{d}$. Risk differences did not vary appreciably over the years of the study (see eFigures 1 and 2 in the Supplement).
Sensitivity Analyses

In 2-adult households ( $\mathrm{n}=2706$ 922), an opioid prescription to 1 adult was associated with increased risk of opioid initiation in the other (RD, 1.08\% [95\% CI, 0.90\%-1.39\%]). Although we controlled for a number of potential confounders, we also assessed the sensitivity of our findings to unmeasured baseline differences between the opioid and NSAID groups (eTable 2 in the Supplement). We found that an unmeasured confounder that is modestly associated with the exposure (eg, prevalence difference $=0.9 \%$ ) and the outcome (eg, RD = 0.9) after adjustment for all measured variables could explain our observed estimate of the RD $(0.71 \%)$. Alternative scenarios are presented in eTable 2 in the Supplement.

\section{Discussion}

We conducted a large, retrospective, cohort study comparing the risk of opioid initiation among commercial insurance beneficiaries who were newly exposed to prescription opioids vs prescription NSAIDs through a household member's prescription. We observed a $0.71 \%$ absolute higher 1-year risk of prescription opioid use among individuals in opioid households compared with prescription NSAID households, which could reflect increased access to these products or unmeasured risk factors. Associations varied across age, region, potential indication, and characteristics of the index prescription.

Previous studies on prescription opioids have used MarketScan, ${ }^{36-39}$ but none examined patterns of use within families. Prior research within families focused on disentangling genetic vs environmental transmission of drug abuse ${ }^{25}$; however, opiate abuse can develop from opioids prescribed for pain management. ${ }^{40}$ Because medication sharing cannot be captured in administrative data, understanding patterns of prescribed opioid use within households may be a critical first step toward addressing the opioid epidemic. If associations across households and social networks are validated in future studies, even small risk differences may be relevant on a population level, considering the broad environmental availability of prescription opioids.

We assessed whether our results could be explained by potential unmeasured differences between members of opioid and NSAID households in sensitivity analyses. Unmeasured variables include whether household members see the same health care professional with a preference for prescribing opioids. We found that the magnitude of such an effect need not be large to explain our findings. A previous study estimated physician preference for prescribing the same analgesic to be more than $20 \%{ }^{41}$; thus, it is plausible that our results could be explained by unmeasured health care professional preference if household members see the same provider (eg, physician, dentist). Alternatively, unmeasured environmental or system-level factors or household socioeconomic status may have given rise to confounding. ${ }^{34}$

The association between potential access to household opioids and opioid initiation could be explained by multiple mechanisms. First, family members may share their medications with other members, which may lead to one seeking his 
Table 2. Cumulative Incidence of Opioid Initiation Among Household Members of Prescription Opioid vs Prescription NSAID Initiators Overall and by Age, Region, and Potential Indication for Opioid Initiation by Patient O, MarketScan Commercial Claims and Encounters, 2000-2014

\begin{tabular}{|c|c|c|c|c|}
\hline \multirow{2}{*}{$\begin{array}{l}\text { Opioid } \\
\text { Initiation }\end{array}$} & \multicolumn{2}{|c|}{ 1-Year Risk, \% $(95 \% \mathrm{CI})^{\mathrm{a}}$} & \multirow{2}{*}{$\begin{array}{l}\text { Risk Difference, } \\
\%(95 \% \mathrm{Cl})^{\mathrm{b}}\end{array}$} & \multirow{2}{*}{$\begin{array}{l}\text { Risk Ratio } \\
(95 \% \mathrm{Cl})\end{array}$} \\
\hline & Opioid & NSAID & & \\
\hline Unadjusted & $11.68(11.66-11.70)$ & $10.60(10.57-10.63)$ & $1.08(1.05-1.11)$ & $1.10(1.10-1.11)$ \\
\hline $\begin{array}{l}\text { Inverse- } \\
\text { probability } \\
\text { weighted }\end{array}$ & $11.83(11.81-11.85)$ & $11.11(11.09-11.14)$ & $0.71(0.68-0.74)$ & $1.06(1.06-1.07)$ \\
\hline \multicolumn{5}{|l|}{ Subgroup analyses } \\
\hline \multicolumn{5}{|l|}{ Age group, y } \\
\hline $0-5$ & $3.6(3.5-3.6)$ & $3.2(3.1-3.2)$ & $0.41(0.35-0.48)$ & $1.13(1.11-1.15)$ \\
\hline $6-11$ & $3.7(3.7-3.8)$ & $3.3(3.3-3.4)$ & $0.41(0.36-0.45)$ & $1.12(1.11-1.14)$ \\
\hline $12-17$ & $10.2(10.2-10.3)$ & $9.5(9.4-9.5)$ & $0.77(0.70-0.86)$ & $1.08(1.07-1.09)$ \\
\hline $18-25$ & $13.7(13.6-13.7)$ & $12.8(12.7-12.8)$ & $0.91(0.81-1.01)$ & $1.07(1.06-1.08)$ \\
\hline $26-35$ & $18.8(18.7-18.9)$ & $17.5(17.4-17.7)$ & $1.26(1.08-1.43)$ & $1.07(1.06-1.08)$ \\
\hline $36-45$ & $16.0(16.0-16.1)$ & $15.3(15.2-15.4)$ & $0.72(0.62-0.80)$ & $1.05(1.04-1.05)$ \\
\hline $46-55$ & $15.7(15.6-15.7)$ & $15.1(15.0-15.1)$ & $0.62(0.52-0.71)$ & $1.04(1.03-1.05)$ \\
\hline$\geq 56$ & $17.1(17.0-17.2)$ & $16.3(16.1-16.4)$ & $0.85(0.73-1.00)$ & $1.05(1.04-1.06)$ \\
\hline \multicolumn{5}{|l|}{ Region } \\
\hline Northeast & $9.6(9.6-9.7)$ & $9.1(9.0-9.1)$ & $0.57(0.47-0.66)$ & $1.12(1.11-1.14)$ \\
\hline $\begin{array}{l}\text { North } \\
\text { Central }\end{array}$ & $11.5(11.4-11.5)$ & $11.0(11.0-11.1)$ & $0.44(0.38-0.51)$ & $1.08(1.07-1.09)$ \\
\hline South & $13.2(13.1-13.2)$ & $12.4(12.3-12.4)$ & $0.76(0.71-0.81)$ & $1.07(1.06-1.08)$ \\
\hline West & $11.2(11.2-11.3)$ & $10.3(10.2-10.3)$ & $0.95(0.88-1.02)$ & $1.07(1.06-1.08)$ \\
\hline \multicolumn{5}{|l|}{$\begin{array}{l}\text { Family history } \\
\text { (Patient 0) }\end{array}$} \\
\hline $\begin{array}{l}\text { Back and } \\
\text { neck pain }\end{array}$ & $12.1(12.0-12.1)$ & $11.3(11.3-11.4)$ & $0.78(0.71-0.85)$ & $1.07(1.06-1.08)$ \\
\hline $\begin{array}{l}\text { No back and } \\
\text { neck pain }\end{array}$ & $11.8(11.8-11.8)$ & $11.0(11.0-11.0)$ & $0.81(0.77-0.84)$ & $1.07(1.07-1.08)$ \\
\hline Fracture & $12.1(12.0-12.2)$ & 11.7 (11.5-11.9) & $0.42(0.21-0.61)$ & $1.04(1.02-1.05)$ \\
\hline No fracture & $11.8(11.8-11.9)$ & $11.0(11.0-11.0)$ & $0.82(0.79-0.85)$ & $1.07(1.07-1.08)$ \\
\hline $\begin{array}{l}\text { Sensitivity } \\
\text { analyses, 2-adult } \\
\text { households }^{c}\end{array}$ & $17.7(17.6-17.9)$ & $16.6(16.5-16.8)$ & $1.08(0.90-1.32)$ & $1.06(1.05-1.08)$ \\
\hline
\end{tabular}

Table 3. Cumulative Incidence of Opioid Initiation Among Household Members of Prescription Opioid vs Prescription NSAID Initiators by Characteristics of Index Prescription, MarketScan Commercial Claims and Encounters, 2000-2014

\begin{tabular}{|lllll}
\hline $\begin{array}{l}\text { Characteristic } \\
\text { of Index Prescription }\end{array}$ & $\begin{array}{l}\text { Opioid Household, } \\
1-\mathrm{y} \text { Risk, } \%(95 \% \mathrm{CI})^{\mathrm{a}}\end{array}$ & $\begin{array}{l}\text { NSAID Household, } \\
1-\mathrm{y} \text { Risk, } \%(95 \% \mathrm{Cl})\end{array}$ & $\begin{array}{l}\text { Risk Difference, } \\
\%(95 \% \mathrm{Cl})\end{array}$ & $\begin{array}{l}\text { Risk Ratio } \\
(95 \% \mathrm{CI})\end{array}$ \\
\hline Overall & $11.8(11.8-11.8)$ & $11.1(11.1-11.1)$ & $0.71(0.68-0.74)$ & $1.06(1.06-1.07)$ \\
\hline Supply, d & & & & \\
\hline$<30$ & $12.0(12.0-12.0)$ & $11.5(12.0-11.5)$ & $0.53(0.49-0.57)$ & $1.05(1.04-1.05)$ \\
\hline$\geq 30^{\mathrm{b}}$ & $12.8(12.7-13.0)$ & $11.7(13.0-11.8)$ & $1.14(0.97-1.34)$ & $1.10(1.08-1.11)$ \\
\hline Refills & & & & \\
\hline No & $11.8(11.8-11.9)$ & $11.4(11.9-11.4)$ & $0.44(0.40-0.47)$ & $1.04(1.03-1.04)$ \\
\hline Yes $^{\mathrm{b}}$ & $13.5(13.5-13.6)$ & $12.1(13.6-12.2)$ & $1.43(1.33-1.53)$ & $1.12(1.11-1.13)$ \\
\hline
\end{tabular}

Abbreviation: NSAID, nonsteroidal anti-inflammatory drugs.

${ }^{a}$ Risks, risk differences, and risk ratios adjusted for household size, household composition, year of cohort entry, region and/or age, sex family history of substance abuse, pain and psychiatric comorbidities, use of scheduled and unscheduled prescription medications, and health care utilization.

${ }^{b}$ For risk difference homogeneity, $P<.001$ for all comparisons.

${ }^{c}$ Households with 2 adults older than $18 \mathrm{y}$.

Abbreviation: NSAID, nonsteroidal anti-inflammatory drugs.

a Risks, risk differences, and risk ratios adjusted for household size, household composition, year of cohort entry, region and/or age, sex family history of substance abuse, pain and psychiatric comorbidities, use of scheduled and unscheduled prescription medications, and health care utilization.

${ }^{\mathrm{b}}$ For RD homogeneity, $P<.001$ for all comparisons. or her own prescription. Second, household opioid use may shape attitudes and norms of opioid use. ${ }^{26}$ Third, spousal correspondence of opioid use may be due to homophily, which is defined as the inclination of similar individuals to associate. ${ }^{42}$ Although we could not disentangle potential mechanisms, interventions that address the opioid epidemic could include initiatives promoting safe medication storage within households and safe disposal of unused medications. Clinicians may need to consider the context within which medications will be used to assess the risks and benefits of prescribing opi- oids. For certain patients, prescribing in smaller quantities or without refills may help mitigate the risk of subsequent opioid use by a household member.

\section{Strengths and Limitations}

We generalized the "new-user" design ${ }^{28}$ to leverage information on households in large administrative databases and restrict to enrollees who had no prior access to opioids or NSAIDs in the household. The benefit of our "new-household" approach is the exclusion of households with prevalent use of 
prescription opioids, which may be affected by differential attrition, adherence, physiologic adaptation, or early adverse effects. ${ }^{28}$ We used households with prescription NSAIDs as an active comparator group to contextualize households with prescription opioids, which have similar indications and minimize potential bias owing to differential allocation of opioids to certain patients. However, restriction to households limits the generalizability of our inferences and applicability to realworld pharmacotherapy decisions. Moreover, MarketScan oversamples the South and has poor coverage of the West; thus, our study may not directly generalize to the US commercially insured population.

Despite our attempt to improve the internal validity of our estimates, we could not verify coresidence of household members or other sources of opioids, such as coresidents with public insurance or another health plan. Misclassification of prior exposure could introduce prevalent user bias, whereas underascertainment of true events will bias the RD even with perfect specificity and nondifferential sensitivity. ${ }^{43}$ Other databases with residential addresses to link patients or with clinician or system characteristics could help address exposure and outcome misclassification.

The cohort and primary outcome were identified using pharmacy dispensing billing claims. Electronic pharmacy dispensing claims are considered the gold standard of prescription drug exposure compared with self-reported medication use or outpatient medical records because insurance reim- bursement is based on complete and accurate claims. ${ }^{44} \mathrm{Al}-$ though opioids paid for in cash or received in inpatient settings were not captured in our data, approximately $15 \%$ of opioid prescriptions in 2008 were paid for in cash $^{45}$ and $90 \%$ of opioids are dispensed from retail pharmacies. ${ }^{46}$ Similarly, pharmacy dispensing billing claims were used to identify prescription NSAIDs. Although we expect the sensitivity of prescription NSAID exposure to be high, we lack information on over-the-counter (OTC) NSAID use. However, OTC NSAID exposure is not expected to be a substantial source of bias in our study because sensitivity analyses have shown that prescription claims data can provide valid estimates of drug-outcome associations even when a large proportion of drug use is OTC. ${ }^{47}$

\section{Conclusions}

Although we cannot rule out shared risk factors, such as clinician preference, prescription drug monitoring, or genetic predilection, our results suggest that opioid prescribing decisions may need to take into account the context within which medications will be used and the potential risk of subsequent opioid initiation by other individuals. Addressing the opioid use epidemic will require comprehensive solutions for all aspects contributing to this public health issue, including the potential for medication sharing and other unintended consequences of prescription opioid use in households.

\section{ARTICLE INFORMATION}

Accepted for Publication: October 22, 2017.

Published Online: December 11, 2017. doi:10.1001/jamainternmed.2017.7280

Author Contributions: Dr Seamans had full access to all of the data in the study and takes responsibility for the integrity of the data and the accuracy of the data analysis.

Study concept and design: Seamans, Carey, Westreich, Cole, Wheeler, Brookhart. Acquisition, analysis, or interpretation of data: Seamans, Wheeler, Alexander, Pate, Brookhart. Drafting of the manuscript: Seamans, Westreich, Brookhart.

Critical revision of the manuscript for important intellectual content: Seamans, Carey, Westreich, Cole, Wheeler, Alexander, Pate, Brookhart. Statistical analysis: Seamans, Westreich, Pate, Brookhart.

Administrative, technical, or material support: Carey, Pate, Brookhart.

Study supervision: Carey, Westreich, Cole, Wheeler Brookhart.

Conflict of Interest Disclosures: Dr Westreich receives research support from the National Institutes of Health (NIH) (DP2 HD084070) and is a scientific advisor to Sanofi-Pasteur on unrelated projects. Dr Wheeler receives research support from Pfizer Inc on unrelated projects. Dr Alexander is Chair of the FDA's Peripheral and Central Nervous System Advisory Committee, is a paid consultant to QuintilesIMS, serves on the Advisory Board of MesaRx Innovations, holds equity in Monument Analytics, and serves as a member of OptumRx's P\&T Committee. This arrangement has been reviewed and approved by the Johns Hopkins
Bloomberg School of Public Health. Dr Brookhart receives investigator-initiated research funding from the NIH (RO1 AG042845; R21 HD080214; RO1 AG023178) and through contracts with the Agency for Healthcare Research and Quality's Developing Evidence to Inform Decision Effectiveness program and the Patient Centered Outcomes Research Institute. Dr Brookhart has received research support from Amgen and AstraZeneca and has served as a scientific advisor for Amgen, Merck, GlaxoSmithKline, UCB BioSciences, RxAnte, and World Health Information Consultants.

Funding/Support: Dr Seamans was supported by the UNC Graduate School Dissertation Completion Fellowship and the National Institutes of Health (T32 CA201159). The database infrastructure used for this project was funded by the Department of Epidemiology, UNC Gillings School of Global Public Health; the Cecil G. Sheps Center for Health Services Research, UNC; the CER Strategic Initiative of UNC's Clinical Translational Science Award (UL1TRO01111); and the UNC School of Medicine.

Role of the Funder/Sponsor: The funding sources had no role in the design and conduct of the study: collection, management, analysis, and interpretation of the data; preparation, review, or approval of the manuscript; and decision to submit the manuscript for publication.

Disclaimer: Data used in this study were obtained under license from the Truven Health Analytics Inc. MarketScan Commercial Claims and Encounters Database (Copyright 2000-2014 Truven Health Analytics Inc. All Rights Reserved).

\section{REFERENCES}

1. Manchikanti L, Helm SII, Fellows B, et al. Opioid epidemic in the United States. Pain Physician. 2012 15(3)(suppl):ES9-ES38.

2. Compton WM, Volkow ND. Major increases in opioid analgesic abuse in the United States: concerns and strategies. Drug Alcohol Depend. 2006;81(2):103-107.

3. Pezalla EJ, Rosen D, Erensen JG, Haddox JD, Mayne TJ. Secular trends in opioid prescribing in the USA. J Pain Res. 2017;10:383-387.

4. Noble M, Tregear SJ, Treadwell JR, Schoelles K. Long-term opioid therapy for chronic noncancer pain: a systematic review and meta-analysis of efficacy and safety. J Pain Symptom Manage. 2008; 35(2):214-228.

5. IMS Institute for Health Care Informatics. The Use of Medicines in the United States: Review of 2011. Parsippany, NJ: IMS Institute for Health Care Informatics; 2012.

6. The use of opioids for the treatment of chronic pain: a consensus statement from the American Academy of Pain Medicine and the American Pain Society. Clin J Pain. 1997;13(1):6-8.

7. Brennan F, Carr DB, Cousins M. Pain management: a fundamental human right. Anesth Analg. 2007;105(1):205-221.

8. Van Zee A. The promotion and marketing of OxyContin: commercial triumph, public health tragedy. Am J Public Health. 2009;99(2):221-227.

9. Joranson DE, Gilson AM, Dahl JL, Haddox JD. Pain management, controlled substances, and state medical board policy: a decade of change. J Pain Symptom Manage. 2002;23(2):138-147. 
10. Hasegawa K, Espinola JA, Brown DF, Camargo CA Jr. Trends in U.S. emergency department visits for opioid overdose, 1993-2010. Pain Med. 2014;15 (10):1765-1770.

11. Braden JB, Russo J, Fan M-Y, et al. Emergency department visits among recipients of chronic opioid therapy. Arch Intern Med. 2010;170(16):14251432.

12. Coben JH, Davis SM, Furbee PM, Sikora RD, Tillotson RD, Bossarte RM. Hospitalizations for poisoning by prescription opioids, sedatives, and tranquilizers. Am J Prev Med. 2010;38(5):517-524.

13. Calcaterra S, Glanz J, Binswanger IA. National trends in pharmaceutical opioid related overdose deaths compared to other substance related overdose deaths: 1999-2009. Drug Alcohol Depend. 2013;131(3):263-270.

14. Centers for Disease Control and Prevention (CDC). Vital signs: overdoses of prescription opioid pain relievers: United States, 1999-2008. MMWR Morb Mortal Wkly Rep. 2011;60(43):1487-1492.

15. Substance Abuse and Mental Health Services Administration. Drug Abuse Warning Network, 2011: National Estimates of Drug-Related Emergency Department Visits. Rockville, MD: Substance Abuse and Mental Health Services Administration; 2013.

16. Gwira Baumblatt JA, Wiedeman C, Dunn JR, Schaffner W, Paulozzi LJ, Jones TF. High-risk use by patients prescribed opioids for pain and its role in overdose deaths. JAMA Intern Med. 2014;174(5): 796-801.

17. Shrank WH. Our bulging medicine cabinets: the other side of medication nonadherence. N Engl J Med. 2011;364(17):1591-1593.

18. Lewis ET, Cucciare MA, Trafton JA. What do patients do with unused opioid medications? Clin J Pain. 2014;30(8):654-662.

19. Johnston LD, O'Malley PM, Bachman JG, Schulenberg JE. Secondary School Students. Vol I. Ann Arbor: Institute for Social Research, The University of Michigan; 2011. Monitoring the Future national survey results on drug use, 1975-2010.

20. Substance Abuse and Mental Health Services Administration. Results From the 2012 National Survey on Drug Use and Health: Summary of National Findings. Rockville, MD: SMA; 2013:13-4795.

21. Goldsworthy RC, Schwartz NC, Mayhorn CB. Beyond abuse and exposure: framing the impact of prescription-medication sharing. Am J Public Health. 2008;98(6):1115-1121.

22. Christakis NA, Fowler JH. The collective dynamics of smoking in a large social network. N Engl J Med. 2008;358(21):2249-2258.
23. Rosenquist JN, Murabito J, Fowler JH, Christakis NA. The spread of alcohol consumption behavior in a large social network. Ann Intern Med. 2010;152(7):426-433, W141.

24. Mednick SC, Christakis NA, Fowler JH. The spread of sleep loss influences drug use in adolescent social networks. PLoS One. 2010;5(3): e9775.

25. Kendler KS, Ohlsson H, Sundquist K, Sundquist J. Within-family environmental transmission of drug abuse: a Swedish national study. JAMA Psychiatry. 2013;70(2):235-242.

26. Rigg KK, Murphy JW. Understanding the etiology of prescription opioid abuse: implications for prevention and treatment. Qual Health Res. 2013;23(7):963-975.

27. Adamson DM, Chang S, Hansen LG. Health Research Data for the Real World: The MarketScan Databases. New York, NY: Thompson Healthcare; 2008.

28. Ray WA. Evaluating medication effects outside of clinical trials: new-user designs. Am J Epidemiol. 2003;158(9):915-920.

29. Pearl J. Causal diagrams for empirical research. Biometrika. 1995;82(4):669-688.

30. Cole SR, Hernán MA. Adjusted survival curves with inverse probability weights. Comput Methods Programs Biomed. 2004;75(1):45-49.

31. Howe CJ, Cole SR, Westreich DJ, Greenland S, Napravnik S, Eron JJ Jr. Splines for trend analysis and continuous confounder control. Epidemiology. 2011;22(6):874-875.

32. CDC compilation of benzodiazepines, muscle relaxants, stimulants, zolpidem, and opioid analgesics with oral morphine milligram equivalent conversion factors, 2016 version. Centers for Disease Control and Prevention; 2016. http://www.pdmpassist.org/pdf/BJA_performance _measure_aid_MME_conversion.pdf. Accessed July 8, 2017.

33. Dowell D, Haegerich TM, Chou R. CDC Guideline for Prescribing Opioids for Chronic Pain: United States, 2016. JAMA. 2016;315(15):1624-1645.

34. VanderWeele TJ. Sensitivity analysis for contagion effects in social networks. Sociol Methods Res. 2011;40(2):240-255.

35. Vanderweele TJ, Arah OA. Bias formulas for sensitivity analysis of unmeasured confounding for general outcomes, treatments, and confounders. Epidemiology. 2011;22(1):42-52.
36. Mack KA, Zhang K, Paulozzi L, Jones C. Prescription practices involving opioid analgesics among Americans with Medicaid, 2010. J Health Care Poor Underserved. 2015;26(1):182-198.

37. Birt J, Johnston J, Nelson D. Exploration of claims-based utilization measures for detecting potential nonmedical use of prescription drugs. J Manag Care Spec Pharm. 2014;20(6):639-646.

38. Logan J, Liu Y, Paulozzi L, Zhang K, Jones C. Opioid prescribing in emergency departments: the prevalence of potentially inappropriate prescribing and misuse. Med Care. 2013;51(8):646-653.

39. Paulozzi LJ, Zhang K, Jones CM, Mack KA. Risk of adverse health outcomes with increasing duration and regularity of opioid therapy. J Am Board Fam Med. 2014;27(3):329-338.

40. Volkow ND, McLellan AT. Opioid abuse in chronic pain: misconceptions and mitigation strategies. N Engl J Med. 2016;374(13):1253-1263.

41. Brookhart MA, Wang PS, Solomon DH, Schneeweiss S. Evaluating short-term drug effects using a physician-specific prescribing preference as an instrumental variable. Epidemiology. 2006;17(3): 268-275.

42. Shalizi CR, Thomas AC. Homophily and contagion are generically confounded in observational social network studies. Sociol Methods Res. 2011;40(2):211-239.

43. Rothman KJ. Modern Epidemiology. Philadelphia, PA: Wolters Kluwer Health/Lippincott Williams \& Wilkins; 2008.

44. Schneeweiss S, Avorn J. A review of uses of health care utilization databases for epidemiologic research on therapeutics. J Clin Epidemiol. 2005;58 (4):323-337.

45. McDonald DC, Carlson KE. The ecology of prescription opioid abuse in the USA: geographic variation in patients' use of multiple prescribers ("doctor shopping"). Pharmacoepidemiol Drug Saf. 2014;23(12):1258-1267.

46. US Department of Justice. Automation of Reports and Consolidated Orders System (ARCOS), Retail Drug Summary. https://www.deadiversion .usdoj.gov/arcos. Accessed January 13, 2017.

47. Yood MU, Campbell UB, Rothman KJ, et al. Using prescription claims data for drugs available over-the-counter (OTC). Pharmacoepidemiol Drug Saf. 2007;16(9):961-968. 